THE Astrophysical JouRnAL, 510:1045-1052, 1999 January 10

(C) 1999. The American Astronomical Society. All rights reserved. Printed in U.S.A.

\title{
MAGNETIC ENERGY RELEASE IN DYNAMIC FAN RECONNECTION MODELS
}

\author{
I. J. D. CRAIG \\ University of Waikato, Hamilton, New Zealand \\ AND \\ A. N. McClymont \\ Institute for Astronomy, University of Hawaii, Honolulu, HI 96822 \\ Received 1997 October 10; accepted 1998 August 7
}

\begin{abstract}
The problem of dynamic, three-dimensional magnetic reconnection is considered. Analytic "fan current" solutions are derived by superposing plane-wave disturbances on magnetic X-point equilibria. The localization of the wave produces a strong current sheet containing the neutral point. It is shown that the classical rate of resistive dissipation in the sheet, namely $W_{\eta} \sim \eta^{1 / 2}$, represents the slowest possible energy-loss rate for the disturbance. The conditions required for fast coronal reconnection are then discussed. It is pointed out that significant "flare-like" energy release may be possible under physically realizable conditions. Moreover, the small length scales associated with the current sheet widths of order $\Delta x \sim \eta^{1 / 2}$ suggest that conditions are probably collisionless close to the neutral point. It is argued that our results are consistent with magnetic reconnection simulations that display "stalling" of the merging rate at small plasma resistivities.
\end{abstract}

Subject headings: MHD — plasmas — Sun: flares — Sun: magnetic fields

\section{INTRODUCTION}

Magnetic energy release by reconnection mechanisms has been intensively studied over the last 30 years. Early twodimensional studies of quasi-steady magnetic merging have highlighted the key role played by near-singular current layers in resistive dissipation (Syrovatskii 1971; Parker 1979). Current sheets accumulate magnetic energy at null points in the plasma, and provide sites for strong magnetic energy release (Forbes \& Priest 1987; Biskamp 1994; McClymont \& Craig 1996a). Recent exact reconnection solutions, however, suggest that current sheets are associated mainly with three-dimensional "fan current" reconnection (Craig \& Fabling 1996). Other reconnection solutions require quasi-cylindrical "spine" currents that develop along the exhaust axis of the fluid (see Galsgaard, Rickard, \& Reddy 1996). Magnetic merging may also involve multiple nulls - as in separator reconnection (e.g., Démoulin et al. 1996).

Yet a major difficulty for any reconnection model is achieving a fast energy dissipation rate in the low-resistivity plasma typical of the solar corona. Despite the development of exact spine and fan solutions, the ability of any reconnection mechanism to explain the massive, explosive magnetic collapse of the solar flare remains questionable. In a recent paper, Craig, Fabling, \& Watson (1997) point out that fan geometries are more favorable than spine geometries for producing flarelike energy release. They argue that all flux pileup solutions - and these seem to be the only exact models with the potential for fast energy release - must eventually stall at some level determined by the plasma resistivity $\eta$. When this is taken into account, the spine energy release saturates long before reaching flare levels. Admittedly, the arguments of Craig et al. (1997) are limited to steady state solutions and mention only briefly effects that are likely to be of considerable importance in the flare context, for instance, the breakdown of classical collisional resistivity. They do suggest, however, that the saturated output of fan reconnection may be sufficient to power modest flares, even when collisional resistivities are assumed.

Fan current reconnection is illustrated schematically in Figure 1. Magnetic shear waves, with motions in the horizontal plane, parallel to the "fan," propagate vertically in the direction of the "spine." The waves converge on the fan plane where they produce an intense current sheet. This leads to strong ohmic dissipation and reconnection of the sheared field across the fan plane. Sufficient amplification of the currents to achieve "fast" reconnection (i.e., a rate that does not fall off as resistivity is decreased) in general requires the waves to be "driven" toward the fan by a strong "external" flow field. Although this flow is imposed a priori on the system considered here, it is a natural consequence of magnetic collapse driven by instability of the coronal field or by emerging flux. The flow is "external" only because our model of the dynamics of the reconnection region has "open" boundaries and so does not encompass the entire system. Alfvénic fluid motions driven by external magnetic forces are, in fact, required for all forms of quasisteady merging (Craig \& Rickard 1994; Priest \& Titov 1996). That is, fluid cannot advect across magnetic separatrices if the flow field is sub-Alfvénic.

The aim of the present paper is to review fan reconnection models within a dynamic, three-dimensional framework. Our starting point is the observation that all current sheet solutions can be viewed as the superposition of transient, nonlinear, plane-wave disturbances on threedimensional magnetic X-points (Craig \& Fabling 1998). These disturbances concentrate in the vicinity of the neutral point and eventually form current sheets that dissipate the accumulated magnetic energy. We demonstrate, for example, how steady state models can be understood as a relaxation of transient wave solutions and show how analytic scaling laws can be derived for the dependence of the ohmic dissipation rate on the plasma resistivity $\eta$. We go on to discuss the energy dissipation characteristics of "fast" solutions, given the likelihood of collisionless conditions 


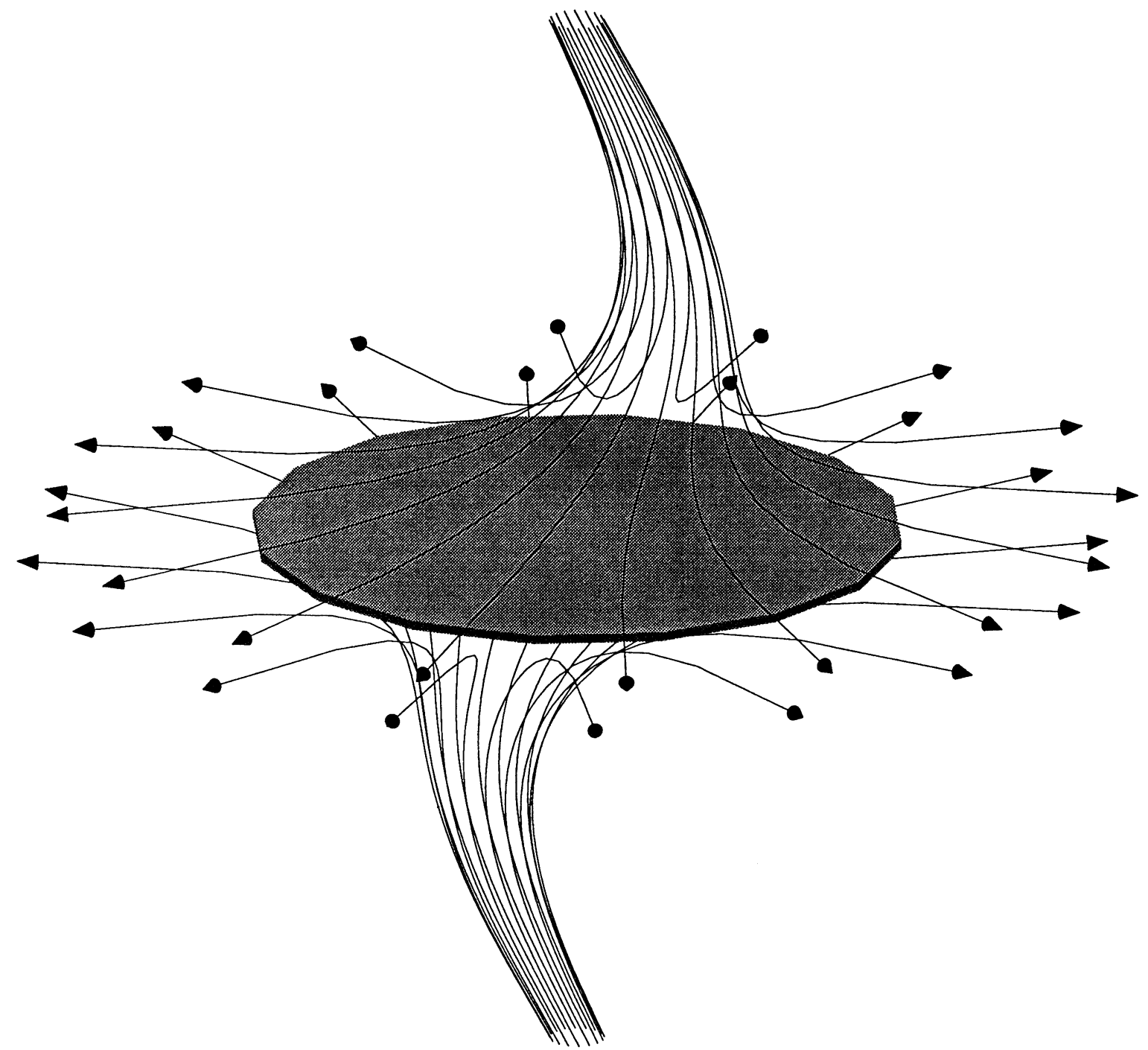

FIG. 1.-Schematic representation of a three-dimensional null point, showing the horizontal "fan" and vertical "spine." A current sheet is formed when vertically propagating waves converge in the fan plane.

near the neutral point.

In $\S 2$ we introduce the governing MHD equations and briefly review the geometry of X-type magnetic equilibria. Section 3 formulates the reconnection solution in terms of plane-wave disturbances impacting on the X-point. These solutions are interpreted in $\S 4$, where a quantitative estimate is made of the saturated energy release under realistic coronal conditions. Our conclusions are presented in $\S 5$.

\section{TIME-DEPENDENT RECONNECTION EQUATIONS}

\subsection{Momentum and Induction Equations}

We consider the nondimensional momentum and induction equations for an incompressible inviscid plasma. An open geometry is assumed in which mass and energy can enter and leave the reconnection region. The reconnection volume is taken to be a cube with $-1 \leq x, y, z \leq+1$.

The governing MHD equations are written in the form

$$
\begin{gathered}
\frac{\partial \omega}{\partial t}+(\boldsymbol{v} \cdot \nabla) \omega-(\omega \cdot \nabla) v=(\boldsymbol{B} \cdot \nabla) \boldsymbol{J}-(\boldsymbol{J} \cdot \nabla) \boldsymbol{B}, \\
\frac{\partial \boldsymbol{B}}{\partial t}+(\boldsymbol{v} \cdot \nabla) \boldsymbol{B}-(\boldsymbol{B} \cdot \nabla) v=\eta \nabla^{2} \boldsymbol{B},
\end{gathered}
$$

where the magnetic and velocity fields satisfy

$$
\boldsymbol{\nabla} \cdot \boldsymbol{B}=0, \quad \boldsymbol{\nabla} \cdot \boldsymbol{v}=0,
$$

and the current density and fluid vorticity are given by

$$
\boldsymbol{J}=\boldsymbol{\nabla} \times \boldsymbol{B}, \quad \omega=\nabla \times \boldsymbol{v} .
$$

The only energy sink for the system is provided by the resistive term. The collisional resistivity $\eta$ is very small-in our dimensionless units it is an inverse Lundquist number of order $10^{-14}$. Thus resistive dissipation can be significant only in localized regions of high current density. It follows that any MHD mechanism significant for reconnection must incorporate a natural, dynamic collapse to small length scales. This collapse in turn may lead to a breakdown of the fluid approximation, as discussed in $\S 4$.

\subsection{Magnetic $X$-Point Equilibria}

Reconnection is generally associated with null points in the magnetic field. We consider an isolated null and expand the magnetic field in the neighborhood of the point $\boldsymbol{r}=\boldsymbol{r}_{0}$ (say) according to

$$
B_{i}\left(\boldsymbol{r}_{0}+\boldsymbol{d r}\right)=B_{i}\left(\boldsymbol{r}_{0}\right)+B_{i, j}\left(\boldsymbol{r}_{0}\right) d x_{j},
$$


where $B_{i, j}$ is an element of the Jacobian matrix $\partial \boldsymbol{B} / \partial \boldsymbol{r}$. The constraint $\boldsymbol{\nabla} \cdot \boldsymbol{B}=0$ then implies the trace condition $B_{i, i}=0$. Obviously, the current density at $x_{0}$, namely,

$$
J_{i}=\epsilon_{i j k} B_{k, j},
$$

vanishes identically only in the case of symmetric $B_{k, j}$. Conversely, nonsymmetric components in $B_{k, j}$ signal the presence of nonpotential nulls.

Now let $\boldsymbol{r}_{0}=0$ locate the null point of the field. The simplest null of interest has the potential form

$$
\boldsymbol{P}(\boldsymbol{r})=[-x \hat{\boldsymbol{x}}+\kappa y \hat{\boldsymbol{y}}+(1-\kappa) z \hat{\boldsymbol{z}}],
$$

where $\kappa$ defines the degree of anisotropy of the field. More general nulls require three extra parameters, specifying the antisymmetric components of $B_{k, j}$ (see e.g., Parnell 1996). However, if force-free conditions $\boldsymbol{J} \times \boldsymbol{B}=0$ are imposed, then two further constraints are introduced. In this case the null is described by only two free parameters.

Returning to the potential (eq. [2.7]), we note that the choice $|\kappa|<1$ assigns a special role to the $x$-axis. This forms a separatrix field line - the spine curve - into the null. The remaining separatrix lines are outgoing and form the "fan" in the plane $x=0$. The special case $\kappa=\frac{1}{2}$ corresponds to rotational symmetry about the $x$-axis. The null degenerates to a planar X-point by taking either $\kappa=0$ or $\kappa=1$.

Finally, we mention that $\boldsymbol{P}$ can also be interpreted as a global velocity field for the plasma. Since we are allowing material to enter and leave the reconnection region, we must account for the possibility of background equilibrium flows. Identifying $\boldsymbol{P}$ as a velocity field, we see that material washed in along the spine is ejected through the outflow surfaces $|y|=1$ and $|z|=1$. In what follows we shall assume that the inflow surfaces $x= \pm 1$ are physically equivalent and consider only the region of positive $x$.

\section{3. $X$-Point Disturbance Fields}

To develop reconnection solutions we superpose some transient magnetic disturbance field $\boldsymbol{Q}$ onto the background potential (eq. [2.7]). In fan current reconnection, the disturbance field is represented by plane waves propagating along the spine axis, specifically

$$
\boldsymbol{Q}_{F}=\boldsymbol{Q}(x, t), \quad \boldsymbol{Q} \cdot \hat{\boldsymbol{x}}=0 .
$$

This form distorts the spine of the background field and leads to strong currents localized in the fan $x=0$. An alternate form, namely,

$$
\boldsymbol{Q}_{S}=Q(y, z, t) \hat{\boldsymbol{x}},
$$

leads to dynamic spine current reconnection, in which quasi-cylindrical currents are aligned to the spine axis. The equations for time-dependent spine reconnection can be found in Craig \& Fabling (1998); they will not be considered here.

Although fan and spine disturbances are mathematically convenient - they satisfy the straight field line condition $(\boldsymbol{Q} \cdot \nabla) \boldsymbol{Q}=0$ - other forms of disturbance field can yield much physical insight. In particular, Hassam (1995), Hassam \& Lambert (1996) and Craig \& McClymont (1997) have considered the behavior of isolated wave packets in the vicinity of magnetic nulls. It is found that only modes which strongly focus toward the null, that is, compressional Alfvén modes, are consistent with fast dissipation: shear modes, unless within a very small capture cross section, exit the reconnection region before significant ohmic dissipation can occur. Indeed, when working in open geometries, the identification of fast ohmic decay can be obscured by shearing wave motions that carry energy out of the source volume (Craig \& McClymont 1997; Craig \& Fabling 1998). In such cases the strength of the current layer (see §3.2) provides a reliable indicator for fast ohmic release.

\subsection{Equations of Fan Current Reconnection}

Let us suppose the fan displacement field $Q(x, t)$ is aligned to some preferred direction, say the $y$-axis. This involves no lack of generality, since the degree of anisotropy of the background field $\boldsymbol{P}$ can still be chosen arbitrarily. We therefore assume the forms

$$
\boldsymbol{v}=\alpha \boldsymbol{P}(\boldsymbol{r})+V(x, t) \hat{\boldsymbol{y}}, \quad \boldsymbol{B}=\beta \boldsymbol{P}(\boldsymbol{r})+Y(x, t) \hat{\boldsymbol{y}},
$$

where $\alpha$ and $\beta$ are positive constants of order unity, and the displacement fields $V(x, t)$ and $Y(x, t)$ are taken as odd functions of $x$. Under these conditions, the momentum and induction equations reduce to

$$
\begin{gathered}
V_{t}=\alpha\left(x V_{x}-\kappa V\right)-\beta\left(x Y_{x}-\kappa Y\right), \\
Y_{t}=\alpha\left(x Y_{x}+\kappa Y\right)-\beta\left(x V_{x}+\kappa V\right)+\eta Y_{x x} .
\end{gathered}
$$

The momentum equation has been integrated by parts and the constant of integration set to zero by virtue of the oddness of the disturbance fields.

By taking $\kappa=0$ or $\kappa=1$ we can derive all the dynamic planar solutions published so far in the literature. We refer to the merging as "driven" if the potential flow field $\alpha>0$ is present. Thus when $\kappa=1$ the diverging flow field is aligned with the wave field $Y(x, t) \hat{\boldsymbol{y}}$ and the field is stretched and amplified; when $\kappa=0$ the wave field is perpendicular to the background flow, and so the field lines are advected incompressibly without magnification (see Fig. 2). We obtain the planar shear wave solutions of Bulanov, Shasharina, \& Pegararo (1990) and the perpendicular shear wave solutions of McClymont \& Craig (1996b) by setting $\alpha=0$ and taking $\kappa=1$ and $\kappa=0$, respectively. The driven annihilation mode of Clark (1964) corresponds to the choice $\beta=V=0$, $\kappa=1$. To obtain the quasi-steady solution of Craig \& Henton (1995) we take $\kappa=1$ and set the time derivatives to zero.

\subsection{The Plasma Pressure}

To recover the plasma pressure we must uncurl the momentum equation. The result is

$$
p(r, t)=p_{0}(t)-\frac{1}{2}\left(\alpha^{2} P^{2}+Y^{2}\right)-\beta \kappa y Y,
$$

where $P=|\boldsymbol{P}(\boldsymbol{r})|$ and $Y=Y(x, t)$. This expression is interpreted in terms of dynamic fan current solutions in $\S 3.1$ and $\S 3.2$ below.

For the present, we note a difficulty with planar models of fast magnetic merging - these require huge pressures in the reconnection region to sustain the dissipation rate (see McClymont \& Craig 1996b; Inverarity \& Priest 1996). Specifically, fast dissipation is associated with "flux pileup" current sheets in which the disturbance field amplitude builds up monotonically with reductions in the plasma resistivity decreases. The increasing hydromagnetic pressure is then reflected by the term $Y^{2} / 2$, which becomes increasingly large as $\eta \rightarrow 0$. 

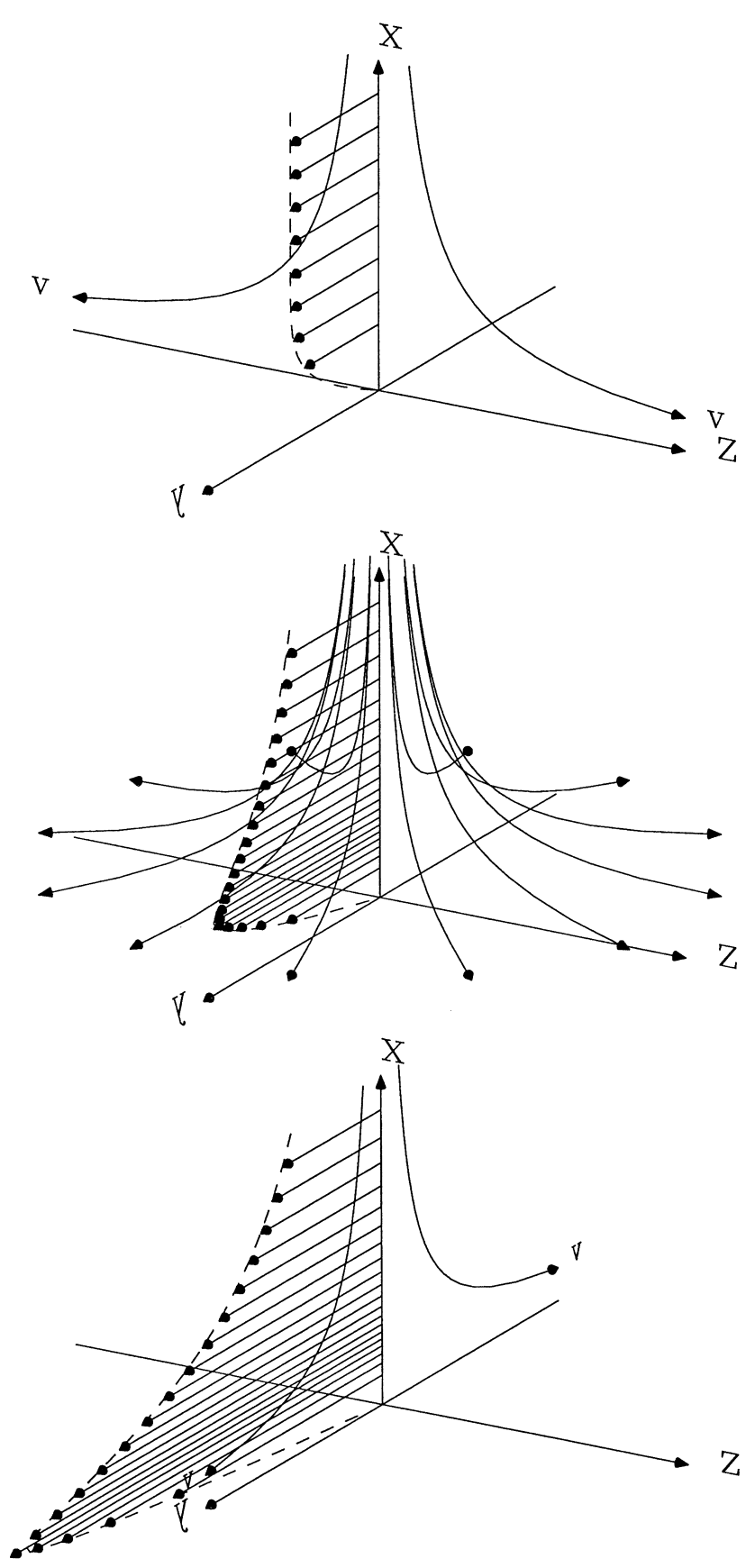

FIG. 2.-Schematic representation of "flux pileup" in response to different background flows. On the left, the background flow for $\kappa=0$ advects the magnetic field incompressibly and does not stretch the field lines. In the middle, isotropic divergence of the flow for $\kappa=1 / 2$ provides the minimum stretching and field amplification necessary for fast dissipation. On the right, maximum stretching of the field lines is obtained for $\kappa=1$.

This problem only arises, as we shall see, when the merging is driven by a strong background flow. Undriven shear waves (Bulanov et al. 1990; McClymont \& Craig 1996b) do not suffer this difficulty, but neither do they produce fast dissipation. (See also the compressible discussion of Hassam \& Lambert 1996). In other words, the ohmic dissipation rate

$$
W_{\eta}=\eta \int J^{2} d V \equiv \eta\left\langle J^{2}\right\rangle
$$

is slow: it scales as a positive power of $\eta$, specifically $W_{\eta} \sim$ $\eta^{1 / 2}$. In contrast, fast-mode solutions show that $Y$ builds up as $\eta^{-1 / 2}$, consistent with superfast dissipation $W_{\eta} \sim \eta^{-1 / 2}$ and the pressure scaling $p_{0} \sim Y^{2} \sim \eta^{-1}$ (Craig \& McClymont 1997). Since such huge hydromagnetic pressures are unrealizable in the solar atmosphere, we must conclude that the buildup of the magnetic field eventually saturates in any realistic fast-merging solution (Craig et al. 1997). The implications of this result are discussed in $\S 4.4$.

\section{DYNAMIC FAN CURRENT SOLUTIONS}

\subsection{Wave Properties of the Solution}

We are interested in the case where some global initial disturbance $\boldsymbol{Q}=Y(x, 0) \hat{\boldsymbol{y}}$ is superposed on the equilibrium $\mathrm{X}$-point. Resistive dissipation will remain negligible until the disturbance has localized to some small length scale determined by $\eta$.

The initial advection phase can be investigated by setting $\eta=0$ and introducing the comoving coordinates

$$
\tau=t, \quad \xi=x e^{\alpha t} .
$$

Eliminating $V(x, t)$ from equation (2.12) yields a KleinGordon equation for $Y(x, t)$, namely,

$$
Y_{\tau \tau}=\beta^{2} Y_{\chi \chi}+\kappa^{2}\left(\alpha^{2}-\beta^{2}\right) Y, \quad \chi=\ln \xi .
$$

This wave equation holds for any linear combination of $Y$ and $V$ and generalizes the planar form $(\kappa=1)$ derived by Craig \& McClymont (1997).

As Craig \& Fabling (1998) have emphasized, the wave properties of the fan reconnection problem can be likened to the one-dimensional, forced oscillations of an elastic medium. The first term in $\beta^{2}$ accounts for magnetic shear waves and reflects the restoring forces in the medium. In fact, the wave equation has two characteristics, namely $C^{ \pm}=\ln \xi \pm \beta \tau$, or in real space,

$$
C^{+}=x e^{-(\alpha+\beta) t}, \quad C^{-}=x e^{-(\alpha-\beta) t}
$$

(see also Craig \& McClymont 1997). The net effect is to split an initial wave packet into two components. One component propagates rapidly inward, but the other can do so only if $\alpha>\beta$, that is, if the driving is sufficiently strong. Since inward-pointing characteristics converge toward each other, initial gradients in the field become magnified, producing strong current densities. Conversely, the current density weakens in any pulse which is outward propagating.

Aside from the localization of the wave envelope for $\alpha>\beta$, there remains the possibility of direct growth in the field amplitude, due to the last (forcing) term in equation (3.2). A necessary condition for growth is clearly

$$
\kappa^{2}\left(\alpha^{2}-\beta^{2}\right)>0 \text {. }
$$

Growth requires $\kappa \neq 0$, plus the field localization condition $\alpha>\beta$.

Is equation (3.4) sufficient for growth? Taking an isolated Fourier mode for the solution yields

$$
Y_{F}(\xi, \tau)=e^{\nu \tau \pm i k \ln \xi}, \quad v(k)=\sqrt{\alpha^{2} \kappa^{2}-\beta^{2}\left(\kappa^{2}+k^{2}\right)},
$$

where $k$ is the wavenumber. Since the space domain in the $\chi=\ln \xi$ variable is noncompact, a global initial disturbance suggests that we choose a very small wavenumber, that is $\dot{k} \ll 1$. More physically, the wave domain corresponds to 
the real space interval $\Delta x \leq x \leq 1$, where $\Delta x$ is the width of the current layer. This suggests that we should choose $k|\ln \Delta x| \simeq 1$ to ensure a global disturbance in the wave domain. As shown below, current sheet widths are so small that equation (3.4) is effectively sufficient for growth.

\subsection{Annihilation of Straight Field Lines}

The case $\beta=0$ provides a degenerate case of the previous theory. The absence of the background X-point field means that wave modes arising through magnetic tension are eliminated. In this case there is no inherent localization of the field, so the merging has to be driven by a background flow. Since straight field lines are advected by the flow toward the fan plane $x=0$, we obtain the dynamic generalization of the Sonnerup \& Priest (1975) steady state annihilation model.

Suppose we consider the annihilation problem as a simple prototype for reconnective merging. We are interested in predicting the width of the current layer, the strength of the current sheet, and the scaling of the ohmic dissipation rate with $\eta$.

Setting $\beta=0$ in equations (2.11) and (2.12) shows that the magnetic and velocity fields completely decouple. In terms of the comoving coordinates (eq. [3.1]), the advective solution for the flow is simply

$$
V(\xi, \tau)=V_{0}(\xi) e^{-\kappa \alpha \tau} .
$$

This flow, being parallel to the field lines, is nonessential. The solution for the field, namely,

$$
Y(\xi, \tau)=Y_{0}(\xi) e^{\kappa \alpha \tau}, \quad \beta=\eta=0,
$$

shows that for $\kappa \alpha$ nonvanishing, the field is strongly amplified as it approaches the neutral line. This magnification, as illustrated schematically in Figure 2, is a manifestation of the stretching of a fluid line element by the background flow. Note that the product $Y V$ is conserved throughout the advection phase.

In the presence of resistivity, the flow solution (eq. [3.6]) remains valid, but the field evolution is modified according to

$$
Y_{\tau}=\kappa \alpha Y+\eta e^{2 \alpha \tau} Y_{\xi \xi},
$$

an equation which is easily solved using transform methods (Clark 1964). For our purposes, it is enough to consider the behavior of an isolated Fourier pulse

$$
Y(\xi, \tau)=A(\tau) \sin \left(k_{0} \xi\right),
$$

where $A(0)=A_{0}$ is arbitrary. The wavenumber $k_{0}$ should be order unity to ensure a global initial disturbance. The field amplitude satisfies

$$
\frac{A_{\tau}}{A}=\kappa \alpha-\eta k_{0}^{2} e^{2 \alpha \tau}
$$

which reduces to the exponential growth of the wave solution (3.7) for $\eta=0$. However, for finite resistivity the growth saturates when $A_{\tau}=0$, which occurs at time

$$
T=\frac{1}{2 \alpha} \ln \frac{\kappa \alpha}{\eta}
$$

on taking $k_{0} \simeq 1$. By this time the point $\xi=1$ on the wave profile has reached the onset of the diffusion layer $x=\xi e^{-\alpha T}=\Delta x \sim \eta^{1 / 2}$, and the field has attained the amplitude $Y \sim \eta^{-\kappa / 2}$. It follows that the current density and annihilation rate scale according to

$$
J \sim \eta^{-(1+\kappa) / 2}, \quad W_{\eta} \simeq \eta J^{2} \Delta x \sim \eta^{1 / 2-\kappa} .
$$

Thus any solution with $\kappa \geq 1 / 2$ is fast. The scaling of the plasma pressure

$$
p_{0} \sim \frac{1}{2} Y^{2} \sim \eta^{-\kappa}
$$

shows that the $\kappa=1 / 2$ axisymmetric solution offers the weakest pressure buildup consistent with fast dissipation. This scaling is a considerable improvement on the fast planar model $p_{0} \sim \eta^{-1}$.

\subsection{Driven Fast Reconnection}

Let us now attempt to construct scaling laws for the case of driven reconnection. We assume $\kappa \neq 0$ and the localization condition $\alpha>\beta$, which provide necessary conditions for growth of the field. In contrast to the annihilation of straight field lines, we cannot assume that $V(x, t)$ always decays with time. Indeed, we know that in quasi-steady merging $V \rightarrow \beta Y(x) / \alpha$, where

$$
Y(x) \equiv Y_{0} \frac{x}{\mu} M\left(\frac{\kappa+1}{2}, \frac{3}{2}, \frac{-x^{2}}{2 \mu}\right), \quad \mu=\frac{\eta \alpha}{\alpha^{2}-\beta^{2}},
$$

and $M$ is the Kummer function (Craig et al. 1997). Thus $V$ is typically Alfvénic, as expected for reconnection solutions (see § 1). The dynamic problem is further complicated by the fact that there are two characteristics for the wave solution (eq. [3.2]). How do these fit into the dynamic reconnection model?

Suppose we introduce (Craig \& Fabling 1998)

$$
F(x, t)=V(x, t)-\frac{\beta}{\alpha} Y(x, t) ;
$$

to measure departures from the quasi-steady solution. Then equations (2.11) and (2.12) give

$$
F_{t}-\alpha^{+} x F_{x}=-\kappa \alpha^{-} F-\frac{\beta}{\alpha} \alpha^{-}\left(x Y_{x}+\kappa Y\right)-\frac{\beta}{\alpha} \eta Y_{x x}
$$

and

$$
Y_{t}-\alpha^{-} x Y_{x}=\kappa \alpha^{-} Y-\beta\left(x F_{x}+\kappa F\right)+\eta Y_{x x},
$$

where

$$
\alpha^{+}=\frac{\alpha^{2}+\beta^{2}}{\alpha}, \quad \alpha^{-}=\frac{\alpha^{2}-\beta^{2}}{\alpha} .
$$

We see that in the case where $\beta \simeq \alpha$ there are disparate timescales for the advection of $F(x, t)$ and $Y(x, t)$. These mimic the fast and slow characteristics of the general wave equation (3.2). In particular, the limit $\beta \rightarrow \alpha$, corresponding to $\alpha^{-} \rightarrow 2(\alpha-\beta)$, involves very slow localization of the field.

For our present purposes it is sufficient to consider equations (3.16) and (3.17) in the case of slow field localization. A more detailed exposition, with numerical examples, given by Craig \& Fabling (1997) shows that the essential argument can be extended to more general situations. If we suppose that $V(x, t)=0$ initially, we must have $F(x, 0) \equiv$ $F_{0}(x)=-\beta Y(x, 0) / \alpha$ at the beginning of the advection phase. The evolution of $F$ is essentially unhindered by gradients in the field since, for $\alpha^{-}$sufficiently small, the field 
cannot advect significantly. It follows that

$$
F(x, t) \simeq F_{0}\left(\xi^{+}\right) e^{-\kappa \alpha^{-} t}, \quad \xi^{+}=x e^{\alpha^{+} t},
$$

and so the rapid localization of $F(x, t)$ is accompanied by a weak decay.

Consider now the field equation (3.17). The evolution occurs on the slow characteristic, so by the time $Y(x, t)$ can advect significantly, $F(x, t)$ has localized to the origin. It follows that

$$
Y(x, t) \simeq Y_{0}\left(\xi^{-}\right) e^{\kappa \alpha^{-} t}, \quad \xi^{-}=x e^{\alpha^{-} t},
$$

describes the growth of the field. This growth will saturate when $\eta Y_{x x}$ becomes comparable to $Y_{t}$. From this condition-or the Fourier mode analysis of $\S 3.2-$ we obtain the localization timescale

$$
T \simeq \frac{1}{2 \alpha^{-}} \ln \frac{\kappa \alpha^{-}}{\eta}
$$

Thus at the time $t \simeq T$, a current sheet has developed with the scalings

$$
\Delta x \sim \eta^{1 / 2}, \quad J \sim \eta^{-(1+\kappa) / 2}, \quad W_{\eta} \sim \eta^{1 / 2-\kappa} .
$$

It follows that fan reconnection leads to fast dissipation only if $\kappa \geq 1 / 2$.

In summary, the reconnection problem has yielded current sheets with the same scaling characteristics as the annihilation solution (eq. [3.12]), at least under the condition of strong driving, $\alpha>\beta$. However, the current sheet develops on the slow characteristic $C^{-}$according to the timescale (eq. [3.20]). Evidently the rapidly localizing $C^{+}$ characteristic is associated with the initial "equalization" of the magnetic and velocity disturbance fields.

\section{APPLICATIONS TO CORONAL ENERGY RELEASE}

\subsection{Introduction}

We have seen that current sheets naturally develop when plane-wave disturbances are superposed on threedimensional magnetic X-points. Fixed inflow speeds (cf. Litvinenko \& Craig 1998) imply that the width of the sheet is universal $-\Delta x \sim \eta^{1 / 2}$ - but whether the dissipation rate is fast depends on the strength and orientation of the background field. Either way, we note that the classical SweetParker rate, namely $W_{\eta} \sim \eta^{1 / 2}$, is formally the slowest that can be achieved with the present solutions.

In fact, there are two major, related difficulties with interpreting the present analysis. The first relates to the probable breakdown of the classical resistivity, the second to the potentially huge hydromagnetic pressures required to sustain fast reconnection (see Jardine \& Allen 1998; Litvinenko \& Craig 1998). To provide context for these problems, we first review coronal constraints on magnetic energy release models.

\subsection{Energy Release Constraints for Coronal Flares}

It is generally believed that the magnetic field in the low corona contains more than enough energy to supply a flare. In fact, to maintain global constraints-helicity, for example - the bulk of the global coronal field should probably be kept intact. This can be naturally achieved by wave mechanisms which provide relatively minor distortions of the ambient field outside the current layer. It is not achieved by magnetic annihilation solutions that destroy the entire field!
To be more concrete, consider a "stressed" coronal field of strength $B_{c}=100 \mathrm{G}$, occupying the volume $V_{c}=L_{c}^{3}$, where $L_{c}=10^{9.5} \mathrm{~cm}$. A reduction of just $1 \mathrm{G}$ yields an output comparable to a modest flare, namely, $2.5 \times 10^{29}$ ergs. To explain a flare, this energy must be released rapidly, on an Alfvénic timescale. An Alfvén speed of $v_{A}=10^{8.5} \mathrm{~cm}$ $\mathrm{s}^{-1}$, corresponding to a plasma of number density of $10^{10}$ $\mathrm{cm}^{-3}$, leads to a timescale $\tau_{A}=L_{c} / v_{A}=10 \mathrm{~s}$. This is roughly 10 times smaller than the typical flare release time of $10^{2} \mathrm{~s}$, which yields a flare releaserate of $2.5 \times 10^{27}$ $\operatorname{ergs~} \mathrm{s}^{-1}$.

Let us calibrate $W_{\eta}$ in units of the global magnetic energy $\left\langle B_{c}^{2} /(8 \pi)\right\rangle$ divided by the Alfvén time $\tau_{A}$. This unit is $10^{30}$ ergs $\mathrm{s}^{-1}$, and so we must have $W_{\eta} \simeq 2.5 \times 10^{-3}$ if the target rate of $2.5 \times 10^{27} \mathrm{erg} \mathrm{s}^{-1}$ is to be met.

\subsection{The Collisional Resistivity}

To apply fan solutions to the energy release problem, we need to assume a value for the dimensionless resistivity $\eta$. In our units the coronal resistivity is

$$
\eta_{c} \equiv \frac{\tau_{\mathrm{A}}}{\tau_{D}}, \quad \tau_{D}=10^{-13} T_{c}^{3 / 2} L_{c}^{2} \mathrm{~s},
$$

where $\tau_{D}$ is the ohmic diffusion time of Spitzer (1962). Taking $T_{c}=10^{6} \mathrm{~K}$ gives

$$
\tau_{D} \simeq 10^{15} \mathrm{~s}, \quad \eta_{c}=10^{-14} .
$$

This implies fan current sheets of thickness

$$
\Delta x_{c}=\eta_{c}^{1 / 2} L_{c} \simeq 10^{2.5} \mathrm{~cm},
$$

which is considerably smaller than the coronal mean free path

$$
\lambda_{c} \simeq 2 \times 10^{4} \frac{T_{c}^{2}}{\eta_{c}} \simeq 2 \times 10^{6} \mathrm{~cm} .
$$

The above parameters suggest that a collisional resistivity is unlikely to be universally valid, at least for coronal plasmas. Since independent estimates suggest that the classical resistivity may be enhanced by a factor of around $10^{5.5}$ under noncollisional conditions (Parker 1979; Forbes 1996), it may be valid to assume the anomalous value

$$
\eta=\eta_{a} \simeq 10^{-8.5} \text {. }
$$

Are we justified in assuming a collisionless resistivity? In our solutions a magnetic wave sweeps inward and localizes in the vicinity of the neutral point. Fluid comoving with the wave will be collisional, since the wave field is strong and particles are constrained to the gyroradius, typically a few centimetres for electrons in a $10^{2} \mathrm{G}$ field. However, because the background X-point field weakens linearly as $x \rightarrow 0$, the plasma trapped between the wave head and the neutral point can become collisionless when the wave is within $\lambda_{c}$ of the origin. Even so, the current sheet is likely to be collisionless only in a quasi-cylindrical region surrounding the spine. Well away from the spine the particles will be constrained to the gyroradius of the "background" coronal field in the fan. Only if this field is negligible can we anticipate a truly collisionless sheet-and then only if the enhanced temperatures in the sheet make $\lambda_{c}$ comparable to $L_{c}$.

These complications make it extremely difficult to form a coherent picture of coronal fan reconnection. Obviously, the dynamics of the current sheet energy release could be considerably affected. With this in mind, we assess the 
plausibility of the model by determining an "effective" coronal resistivity based on meeting the energy release constraints of $\S 4.2$ above.

\subsection{The Saturated Energy Flux}

Let us estimate the saturated energy dissipation based on an effective coronal resistivity $\eta=\eta_{e}$. For definiteness, we concentrate on the $\kappa \simeq 1 / 2$ solutions, which give, at least formally, the slowest fast reconnection rates (eq. 3.3). We must limit the wave amplitude on the inflow boundary $x=1$ to maintain physically realistic values of the flux pileup field in the sheet, in the manner discussed by Craig et al. (1997) and Watson \& Craig (1997a, 1997b). Although it is possible to obtain an optimum scaling by simultaneously saturating the dynamic and magnetic pressures in the reconnection region (see Litvinenko \& Craig 1998), we shall not consider this refinement here. Specifically, we assume that the sheet field satisfies $Y_{s} \leq 10$, which (being normalized to $B_{c}=100 \mathrm{G}$ ) corresponds to driving the merging by, at most, a $10^{3} \mathrm{G}$ external field. Hydromagnetic pressures 10 times greater than this sheet pressure are certainly available in the lower solar atmosphere $(B \leq 3000 \mathrm{G})$, and we know that pressure enhancements, by factors of 100 or more, are required in flaring loops (Feldman 1996).

Taking a quasi-steady approximation for the field (eq. 3.14) gives the saturated output rate (Craig et al. 1997),

$$
W_{\eta_{e}} \simeq \eta_{e}^{1 / 2} Y_{s}^{2},
$$

and the total energy released

$$
\left\langle\frac{1}{2} Y_{s}^{2}\right\rangle \simeq \eta_{e}^{1 / 2} Y_{s}^{2} T_{D}\left(\eta_{e} ; \kappa\right),
$$

where, based on the $\alpha=1$ asymptotics of equation (3.14),

$$
T_{D}(\eta ; \kappa) \simeq \frac{1}{2} \begin{cases}\frac{1}{3}+\frac{1}{2} \ln \frac{1}{\eta} & \text { if } \kappa=1 / 2 \\ \frac{1}{3}+\frac{1}{(1-2 \kappa)}\left(\eta^{\kappa-1 / 2}-1\right) & \text { otherwise } .\end{cases}
$$

The expression for $T_{D}$ reinforces the fact that the decay is fast for $\kappa \geq 1 / 2$ but becomes slow as $\kappa$ is further reduced. In particular, as $\kappa \rightarrow 0$ we approach the Sweet-Parker result $T_{D} \sim \eta^{-1 / 2}$.

We now equate $W_{\eta_{e}}$ with our target release rate of $2.5 \times 10^{-3}$. For $Y_{s}=10$ this gives

$$
\eta_{e} \simeq 6 \times 10^{-10}
$$

a value which satisfies $\eta_{c}<\eta_{e}<\eta_{a}$. For $\kappa=1 / 2$ this corresponds to washing in a displacement field of just $6 \mathrm{G}$ through the inflow boundary. The total energy released is then $\left\langle\frac{1}{2} Y_{s}^{2}\right\rangle \simeq 1.5 \times 10^{-2}$, which translates to $1.5 \times 10^{29}$ ergs over $T_{D} \simeq 1 / 4\left|\ln \eta_{e}\right| \simeq 5.5$ Alfvén times.

How do these values change as $\kappa$ is varied? Note that as $\kappa$ is reduced, a larger disturbance field is washed through the inflow boundary: this is because we have constrained the peak field $Y_{s}$, and the field falls off behind the wave head increasingly slowly, as $Y \sim x^{-\kappa}$, as $\kappa$ is reduced (see Fig. 2). It follows that the increased decay time as $\kappa \rightarrow 0$ reflects the increasing energy in the outer wave field rather than a slowing of the ohmic dissipation rate in the sheet. Even a formally slow rate, corresponding to $\kappa=0.4$, may still provide an acceptable dissipation time. In this case over $5 \times 10^{29}$ ergs are released in just under 20 Alfvén times.

Returning to the effective resistivity (eq. [4.8]), we note that although $\eta_{e}$ is 5 times smaller than $\eta_{a}$, it is based on the assumption that the dissipation occurs uniformly over a sheet of area $L_{c}^{2}$; however, as discussed in $\S 4.3$, it is likely that the sheet is collisionless over only a small fraction of this area in the region surrounding the neutral point. The effects of a hybrid resistivity on the dynamics of the energy release are as yet unknown.

What happens if we adopt the collisional resistivity (eq. [4.2])? In this case, the energy release target can be achieved only with sheet fields exceeding $10^{4} \mathrm{G}$. Since these are considerably larger than sunspot fields at the base of the solar atmosphere, we can effectively overrule a purely collisional mechanism. Conversely, by taking the anomalous value (eq. [4.5]), the field at the sheet can be reduced to approximately $650 \mathrm{G}$ and still meet the target release rate.

\subsection{Stalling in Magnetic Reconnection Simulations}

A key feature of the fan reconnection solutions is that the wave amplitude on the boundary can be chosen to provide a significant energy release while satisfying the "external" constraint of a plausible hydromagnetic pressure. Waves of too large an amplitude cannot be dissipated rapidly, for these produce pressures in the reconnection region that cannot be sustained by the external hydromagnetic forces available to drive the merging. In other words, the reconnection "stalls" when the external hydromagnetic pressure is exceeded by the magnetic pressure built up in the sheet.

As far as we are aware, this interpretation is borne out by all self-consistent merging computations carried out at sufficiently low resistivities. Biskamp \& Welter (1980) provide anecdotal evidence in the case of the coelescence instability, as do DeLuca \& Craig (1992) for an incompressible, two dimensional simulation in fully periodic geometry. Notably, Henton (1996) has revisited the DeLuca \& Craig (1992) computation using various levels of compressibility. Henton finds that the merging stalls when the pressure of the flux pileup sheet becomes comparable to the magnetic pressure on the boundary (see Craig, Henton, \& Rickard 1993). Even pressureless perturbed X-points show a saturation of flux pileup (McClymont \& Craig 1996a). In this case however, the reconnection rate remains fast by virtue of an acceleration in the thinning of the sheet width, $\Delta x \rightarrow \eta$, as opposed to the customary $\eta^{1 / 2}$ dependency, at least for nonoptimized inflow speeds (see Litvinenko \& Craig 1998). This behavior highlights a key result: that fast reconnection without flux pileup requires a much stronger collapse to small length scales, namely $\Delta x \sim \eta$. To our knowledge there is no self-consistent model, incorporating finite gas pressure, of such an extreme collapse.

\section{CONCLUSIONS}

We have considered dynamic models for fan current reconnection in three space dimensions. We have shown that solutions can be constructed by superposing global, plane wave disturbances on background X-point equilibria. The initial evolution of the disturbance is governed by the Klein-Gordon wave equation and meets a central requirement of any plausible reconnection model - it provides a rapid collapse to small length scales. In fact, a universal feature of all our fan solutions is the development of current sheets of width $\Delta x \sim \eta^{1 / 2}$ overlying the magnetic neutral point.

To provide fast reconnection, however, requires flux pileup in the current layer. If the flux pileup is weak, only modest enhancements over the classical slow rate $W_{\eta} \sim \eta^{1 / 2}$ 
can be achieved. We have noted that flux pileup current layers seem a natural outcome of self-consistent magnetic reconnection simulations (e.g., Biskamp 1994), and even plasma laboratory experiments (Syrovatskii 1981). In the present wave solutions, flux pileup requires a sufficiently strong, suitably oriented, background velocity field. Such flows imply large hydromagnetic pressures throughout the reconnection region. Although the pressure buildup is considerably eased in fully three-dimensional solutions, the reconnection must eventually saturate, depending on the level of the resistivity and the amplitude of the wave field on the inflow boundary. That is, the reconnection stalls whenever the pressure in the sheet exceeds the external hydromagnetic pressure available to drive the merging.

Given that stalling appears as a natural outcome of flux pileup solutions, we have estimated the "saturated" output power of fan reconnection models. This is achieved by limiting the pressure at the neutral point to hydromagnetic pressures realizable within the solar atmosphere. The conditions we adopt, equivalent to a global pressure enhancement of 2 orders of magnitude in the flare, seem within the current observational constraints (see Feldman 1996). Aside from the pressure in the sheet, the saturated output depends only on the plasma resistivity $\eta$. One complication is the likeli- hood of collisionless conditions near the center of the sheet. Based on collisional conditions, we predict current sheet widths of $\Delta x \simeq 10^{2.5} \mathrm{~cm}$, well below the mean free path of the plasma $\left(\lambda_{c} \simeq 10^{4} \Delta x\right)$. Even so, given an appreciable background field in the fan - as required for merging curved field lines - the current sheet is unlikely to be collisionless over its entire area.

Faced with these difficulties we have estimated an "effective" resistivity based on satisfying the output requirements of a typical coronal flare. The saturated power estimate provides an effective resistivity $\eta_{e} \sim 6 \times 10^{-10}$, which is bracketed by the collisional resistivity $\eta_{c}=10^{-14}$ and the plausible anomalous value $\eta_{a}=10^{-8.5}$ (see $\S 4.4$ ). Such estimates are not definitive, but they do suggest that flarelike energy release lies within the compass of fan reconnection models. By contrast, fan models based on collisional coronal resistivities appear to be effectively overruled. Aside from the theoretical implausibility of sheet widths approaching $1 \mathrm{~m}$, the collisional model with $\eta_{c}=$ $10^{-14}$ evidently requires pressures in the sheet well beyond those available in even the strongest sunspot fields.

This work was supported by grant ATM 96-19441.
Biskamp, D. 1994, Phys. Rep., 4, 237

Biskamp, D., \& Welter, H. 1980, Phys. Rev. Lett., 44, 1069

Bulanov, S. V., Shasharina, S. G., \& Pegararo, F. 1990, Plasma Phys. Controlled Fusion, 32(5), 377

Clark, A. 1964, Phys. Fluids, 7(8), 1299

Craig, I. J. D., \& Fabling, R. B. 1996, ApJ, 462, 969

, 1998, Phys. Plasmas, 5, 635

Craig, I. J. D., Fabling, R. B., Henton, S. M., \& Rickard, G. J. 1995, ApJ, 455, L197

Craig, I. J. D., Fabling, R. B., \& Watson, P. G. 1997, ApJ, 485, 383

Craig, I. J. D., \& Henton, S. M. 1995, ApJ, 450, 280

Craig, I. J. D., Henton, S. M., \& Rickard, G. J. 1993, A\&A, 267, L39

Craig, I. J. D., \& McClymont, A. N. 1997, ApJ, 481, 986

Craig, I. J. D., \& Rickard, G. J. 1994, A\&A, 287, 261

DeLuca, E. E., \& Craig, I. J. D. 1992, ApJ, 390, 679

Démoulin, P., Mandrini, C. H., van Driel-Gesztely, L., Priest, E. R., Henoux, J. C., \& Schmieder, B. 1996, in ASP Conf. Ser. 111, Magnetic Reconnection in the Solar Atmosphere, ed. R. D. Bentley \& J. T. Mariska (San Francisco: ASP), 49

Feldman, U. 1996, in ASP Conf. Ser. 111, Magnetic Reconnection in the Solar Atmosphere, ed. R. D. Bentley \& J. T. Mariska (San Francisco: ASP), 145

Forbes, T. G. 1996, in ASP Conf. Ser. 111, Magnetic Reconnection in the Solar Atmosphere, ed. R. D. Bentley \& J. T. Mariska (San Francisco: ASP), 259

Forbes, T. G., \& Priest, E. R. 1987, Rev. Geophys. J., 25, 1583

\section{REFERENCES}

Galsgaard, K., Rickard, G. J., Reddy, R. V., \& Nordland, A. 1996, in ASP Conf. Ser. 111, Magnetic Reconnection in the Solar Atmosphere, ed. R. D. Bentley \& J. T. Mariska (San Francisco: ASP), 82

Hassam, A. B. 1995, Phys. Plasmas, 2, 4662

Hassam, A. B., \& Lambert, R. P. 1996, ApJ, 472, 832

Henton, S. M. 1996, Magnetic Reconnection in Astrophysical Plasmas, Ph.D. thesis, Univ. Waikato

Inverarity, G. W., \& Priest, E. R. 1996, in ASP Conf. Ser. 111, Magnetic Reconnection in the Solar Atmosphere, ed. R. D. Bentley \& J. T. Mariska (San Francisco: ASP), 296

Jardine, M., \& Allen, H. R. 1998, Sol. Phys., 151, 255

Litvinenko, Yu. E., \& Craig, I. J. D. 1998, ApJ, submitted

McClymont, A. N., \& Craig, I. J. D. 1996a, ApJ, 466, 487

, 1996b, in ASP Conf. Ser. 111, Magnetic Reconnection in the Solar Atmosphere, ed. R. D. Bentley \& J. T. Mariska (San Francisco: ASP), 268

Parker, E. N. 1979, Cosmical Magnetic Fields (Oxford: Clarendon Press)

Parnell, C. E. 1996, in ASP Conf. Ser. 111, Magnetic Reconnection in the Solar Atmosphere, ed. R. D. Bentley \& J. T. Mariska (San Francisco: ASP), 19

Priest, E., \& Titov, V. S. 1996, Philos. Trans. R. Soc., London, A, 354, 2951

Sonnerup, B. U. O.., \& Priest, E. R. 1975, J. Plasma Phys., 14, 283

Syrovatskii, S. I. 1971, Sov. Phys., J. Exp. Theor. Phys., 33, 933 , 1981, Annu. Rev. Astron. Astrophys., 19, 163

Watson, P. G., \& Craig, I. J. D. 1997a, Phys. Plasmas, 4, 101 , 1997b, Phys. Plasmas, 4, 110 